\title{
Evaluation of the sensitivity and specificity of a ligase chain reaction test kit for the detection of Chlamydia trachomatis
}

\author{
N Miyashita, A Matsumoto, Y Niki, T Matsushima
}

\begin{abstract}
The sensitivity and specificity of a newly developed ligase chain reaction (LCR) test kit were examined by the use of highly purified elementary bodies (EBs) and in situ inclusions containing reticulate bodies only. The performance of the LCR kit was compared with a commercially available polymerase chain reaction (PCR) test kit, AMPLICOR Chlamydia trachomatis. The number of EBs and inclusions of $C$ trachomatis, respectively, at the detection limit of both kits were two EBs and one inclusion per assay. Neither kit crossreacted with $C$ pneumoniae, $C$ psittaci and $C$ pecorum EBs or reticulate bodies.

(F Clin Pathol 1996;49:515-517)
\end{abstract}

Keywords: Chlamydia trachomatis, ligase chain reaction.

Chlamydia trachomatis is the cause of a large array of sexually transmitted diseases (STDs), as well as ocular infections. Recent epidemiological studies have revealed that $C$ trachomatis is the major cause of STDs such as nongonococcal urethritis, cervicitis and many related diseases, especially in well developed countries. ${ }^{1}$ Given its importance, a number of commercially available test kits for detecting $C$ trachomatis in clinical specimens have been developed and are used routinely for the diagnosis of $C$ trachomatis infection. Among these test kits, the polymerase chain reaction (PCR) test kit for detecting $C$ trachomatis specific 7.5 kilobase plasmid DNA has revolutionised diagnostic sensitivity and specificity. ${ }^{23}$

$C$ trachomatis, as well as other members of the genus Chlamydia, replicate through a unique developmental cycle that involves serial conversion of two distinct forms, the elementary body (EB) and the reticulate body (RB). After internalisation of the EB into the host cell, this extracellular form transforms into the $\mathrm{RB}$, multiplies by binary fission, then subsequently converts back into the EB form. The EBs are uniform in size (about $0.3 \mu \mathrm{m}$ in diameter) but the RBs range in size from 0.5 to $1 \mu \mathrm{m}$, occasionally reaching more than $2 \mu \mathrm{m}$ in diameter. This strongly suggests that the target of the test kits is constant in EBs, but not RBs. Based on this supposition, we have examined the sensitivities of many test kits, such as MicroTrak (Syva, Palo Alto, California, USA), ${ }^{4}$ IDEIA Chlamydia (Dako, Ely, Cambs, UK), ${ }^{45}$ Gen-probe PACE 2 (Gen Probe, San Diego, California, USA), ${ }^{45}$ and the PCR test kit, AMPLICOR $C$ trachomatis (Roche Diag- nostics, Branchburg, New Jersey, USA). ${ }^{34}$ Using the same methodology, we have also examined the sensitivity and specificity of a newly developed ligase chain reaction (LCR) test kit (Abbott, Chicago, Illinois, USA) targeting the 7.5 kilobase plasmid of $C$ trachomatis. ${ }^{67}$

\section{Methods}

The following chlamydial strains were used: $C$ trachomatis $\mathrm{D} / \mathrm{UW}-3 / \mathrm{Cx}, \mathrm{E} / \mathrm{UW}-5 / \mathrm{Cx}, \mathrm{L} 2 / 434 /$ $\mathrm{Bu}$, and 20 clinical isolates; $C$ pneumoniae TW-183, AR-39, AR-388, IOL-207, Kajaani-6, YK-41, KKpn-15, and 14 clinical isolates; $C$ psittaci Prt/GCP-1, Or/B577, Frt$\mathrm{Hu} / \mathrm{Cal}$ 10, Bud/Budgerigar-1, Bud/IZAWA-1, $\mathrm{Hu} / 30 \mathrm{~A}$, and three clinical isolates; and $C$ pecorum $\mathrm{Bo} / \mathrm{E} 58$. The species of all clinical isolates of $C$ trachomatis and $C$ pneumoniae was determined by iodine staining and immunofluorescent staining with MicroTrak and RR402 (Washington Research Foundation, USA) monoclonal antibody specific for $C$ pneumoniae. The propagation of all strains was performed according to previously reported methods. ${ }^{45}$ The purification of EBs was also carried out by a previously reported method, ${ }^{5}$ except that the Renografin gradient centrifugation was repeated three times in order to enhance the purity of the EB preparations. Clinical isolates were tested without purification for chlamydial organisms and therefore contained a lot of cell debris.

Due to their fragility, it was difficult to purify intact RBs of all strains used. Therefore, we prepared appropriate strain infected cells at less than 1 inclusion forming unit (IFU)/cell, harvesting 15 and 19 hours post-inoculation in order to test reactivity against $\mathrm{RBs}$ contained in the inclusions, as described previously. ${ }^{3}$ The number of EBs and inclusion bearing cells was counted by previously reported methods. ${ }^{3}$ Briefly, the purified EB suspension was appropriately diluted and spun down on a coverslip. After shadowcasting, intact EBs were counted in 30 fields of a scanning electron microscope $(\times 8000)$. Based on the count, duplicate series of a twofold dilution were prepared. The number of inclusions in $10^{5}$ cells was counted by flow cytometry (FACStar, Becton Dickinson, USA) after first being prepared by a direct immunofluorescent antibody staining method with a genus specific monoclonal antibody, Cultureset (Ortho Diagnostic Systems), and then being diluted 10-fold in series, in duplicate, as for the EB suspension. To compare the sensitivities of the PCR and LCR kits, each suspension in the dilution series was 

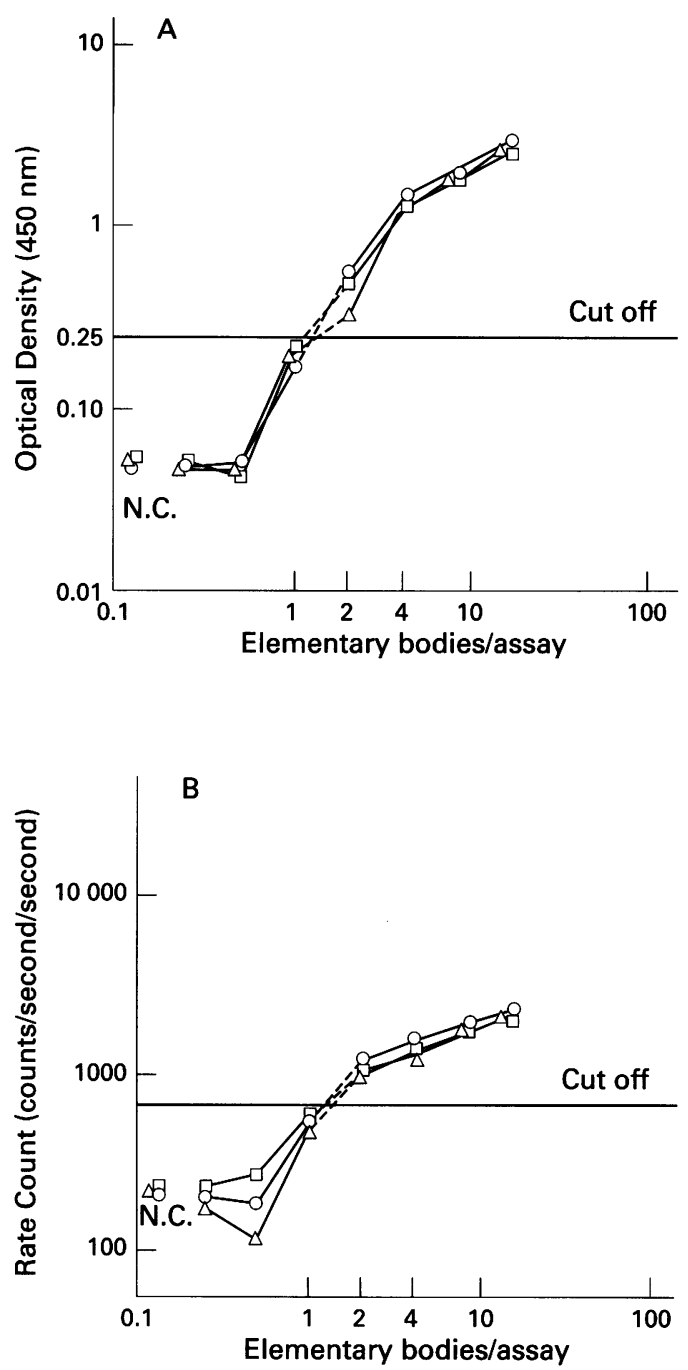

Figure 1 Relation between the particle count of purified $E B$ s of $C$ trachomatis $L 2 / 434 / B u$ and the signal intensity of the (A) PCR and (B) LCR test kits. NC = negative control.

mixed with 10 times the volume of transport medium provided in the PCR kit. Similarly, the extraction medium in the LCR kit was added to the other series of EB and inclusion bearing cell suspensions in order to release the target 7.5 kilobase plasmid DNA. Thereafter, all steps in both tests were carried according to the manufacturers' instructions.

\section{Results}

Electron microscopy, as performed in our previous study, ${ }^{5}$ demonstrated that the newly prepared EB fractions of the $C$ trachomatis, $C$ psit-

Table 1 Reactivities of $\mathrm{C}$ trachomatis inclusions containing RBs only 15 and 19 hours post-inoculation in the PCR and LCR test kits

\begin{tabular}{|c|c|c|c|c|}
\hline \multirow[b]{2}{*}{$\begin{array}{l}\text { Inclusion number } \\
\text { per assay }\end{array}$} & \multicolumn{2}{|l|}{$P C R$} & \multicolumn{2}{|l|}{$L C R$} \\
\hline & $\begin{array}{l}15 \\
\text { hours }\end{array}$ & $\begin{array}{l}19 \\
\text { hours }\end{array}$ & $\begin{array}{l}15 \\
\text { hours }\end{array}$ & $\begin{array}{l}19 \\
\text { hours }\end{array}$ \\
\hline $1 \times 10^{4}$ & + & + & + & + \\
\hline $1 \times 10^{3}$ & + & + & + & + \\
\hline $1 \times 10^{2}$ & + & + & + & + \\
\hline $1 \times 10$ & + & + & + & + \\
\hline 1 & + & + & + & + \\
\hline 0 & - & - & - & - \\
\hline NC & - & - & - & - \\
\hline
\end{tabular}

Symbols: - = no reaction; $+=$ reaction; $\mathrm{NC}=$ negative control. taci and $C$ pecorum strains consisted of almost $100 \%$ intact EBs. However, the EB suspensions of the $C$ pneumoniae strains contained 97-98\% intact EBs and also a few EB envelopes.

The results obtained in several preliminary tests of the LCR kit showed that the EB number at the detection limit ranged from two to four EBs per assay; however, the cut off level (detection limit) was changed from 339 to 400 counts/second/second, as this level must be determined every time an assay is run in this test system. Therefore, in the present study, we assayed all samples at the same time to compare the sensitivities of the PCR and LCR kits accurately.

Figure 1 shows the relation between the number of EBs and the intensity of the signal produced by the PCR (fig 1A) and LCR (fig 1B) kits. The cut off level clearly occurs between one and two EBs per assay (broken lines) in the PCR kit, in agreement with our previous study. ${ }^{3}$ An identical result was obtained with the LCR kit for three different series of diluted samples. We therefore conclude that the sensitivities of the PCR and LCR kits are equivalent and that the newly developed LCR test kit can detect two or more EBs per assay. We emphasise that, based on identical PCR sensitivities in the present and previous studies, the LCR sensitivity obtained from the same dilution series is reliable. No cross-reaction with the EBs of $C$ pneumoniae, $C$ psittaci and $C$ pecorum strains was observed for any of the EB concentrations used (data not shown), indicating that the LCR and PCR kits are highly specific for $C$ trachomatis.

Table 1 shows the number of inclusions detected by the PCR and LCR test kits. One inclusion of $C$ trachomatis was detected by both assays irrespective of the harvest time after inoculation, indicating that RBs could also be detected by both test kits, although the number of RBs at the detection limit was not determined. As for the EBs of $C$ pneumoniae, $C$ psittaci and $C$ pecorum, the RB inclusions of these species were not detected by either kit.

All clinical $C$ trachomatis isolates containing cell debris were also detected with the LCR and PCR kits. This indicates that these kits are applicable to specimens containing cell debris, such as clinical swabs. None of the clinical isolates of other species was detected with either test kit (data not shown).

\section{Discussion}

The target of the LCR test kit and the PCR test kit, AMPLICOR $C$ trachomatis, is the $C$ trachomatis specific 7.5 kilobase plasmid. ${ }^{8}$ We previously reported that the number of EBs at the detection limit (or cut off level) determined by the PCR kit was two to four per assay. ${ }^{3}$ In the present study, an identical result was obtained, confirming the accuracy of our particle counting method applied to purified EBs. The samples tested with the LCR kit were the same as those tested with the PCR kit, and the EB particle number at the detection limit of the LCR test was also identical with that of the PCR test. Therefore, we conclude that the 
sensitivity of the LCR kit is identical with that of the PCR kit and that the LCR kit can detect two or more EBs per assay. The average number of plasmids in one EB was estimated to be about $10 .^{9}$ These results, therefore, indicate that 20 copies or more of the plasmid can be amplified and detected by the LCR kit under the conditions used. Dille $e t a l^{6}$ reported that the sensitivity of the LCR kit which targets the major outer membrane protein gene and the plasmid appeared to be three EBs per assay using purified EBs which were counted by optical microscopy with Giemsa staining. Their results lead us to wonder whether the accuracy of the particle counting, together with the purity of the EB fraction used in their tests, were sufficient for this highly sensitive assay.

$C$ psittaci has also been reported to contain plasmids, ${ }^{10}$ but they were not amplified in either of the assays used in the present study, indicating the sequence differences between $C$ trachomatis and $C$ psittaci. No evidence was obtained for the presence of plasmids in $C$ pneumoniae organisms isolated from humans.

In conclusion, the newly developed LCR test kit, which can be used as a non-radioactive method, is extremely sensitive and specific for the detection of $C$ trachomatis organisms. The manipulation procedures for this assay are simple and designed to minimise carry-over contamination which can cause false positive results. Therefore, we recommend the LCR and PCR kits for the routine diagnosis of $C \mathrm{tra}$ chomatis infection.

This work was supported by Grants-in-Aid of Scientific Research from the Ministry of Education, Science and Culture Research from the Ministry of Education, Science and Culture, Japan (63570204) and
Medical School (6-506).

1 Cates W Jr, Wasserheit JN. Genital chalmydial infections epidemiology and reproductive sequelae. Am $\mathcal{f}$ Obste Gynecol 1991;164:1771-81.

2 Loeffelholz MJ, Lewinski CA, Silver SR, Purohit AP, Herman SA, Buonagurio DA, et al. Detection of Chlamydia trachomatis in endocervical specimens by polymerase chain reaction. $\mathcal{F}$ Clin Microbiol 1992;30:2847-51.

3 Miyashita N, Iijima Y, Matsumoto A. Evaluation of the sensitivity and specificity of polymerase chain reaction test kit, AMPLICOR Chlamydia trachomatis. Microbiol Immunol 1994;38:81-85.

4 Miyashita N, Matsumoto A. Microbiology of chlamydiaewith emphasis on physicochemistry, antigenicity and drug with emphasibility of Chlamydia pneumoniae-. Kawasaki Med $\mathcal{f}$ susceptibility of

5 Miyashita N, Matsumoto A. Establishment of a particlecounting method for purified elementary bodies of chlamydiae and evaluation of sensitivities of the IDEIA Chlamydia kit and DNA probe by using the purified elementary bodies. F Clin Microbiol 1992;30:2911-16.

6 Dille BJ, Butzen CC, Birkenmeyer LG. Amplification of Chlamydia trachomatis DNA by ligase chain reaction. $\mathcal{F}$ Clin Microbiol 1993;31:729-31.

7 Schachter J, Stamm WE, Quinn TC, Andrews WW, Burczak JD, Lee H. Ligase chain reaction to detect Chlamydia traJD, Lee H. Ligase chain reaction to detect Chlamydia tra1994;32:2540-43.

8 Sriprakash KS, Macavoy ES. Characterization and sequence of a plasmid from the trachoma biovar of Chlamydia trachomatis. Plasmid 1987;18:205-14.

9 Palmer L, Falkow S. A common plasmid of Chlamydia trachomatis. Plasmid 1986;16:52-62.

10 Lovett M, Kuo C-C, Holmes K, Falkow S. Plasmids of the genus Chlamydia. In: Nelson JD, Grassi C, eds. Curren chemotherapy and infectious disease, Vol 2. Washington, DC American Society for Microbiology, 1980:1250-2.

\title{
Caseous necrosis in cutaneous leishmaniasis
}

\author{
E Peltier, P Wolkenstein, M Deniau, E-S Zafrani, J Wechsler
}

\begin{abstract}
Service d'Anatomie et de Cytologie

Pathologiques,

Hôpital Antoine

Béclère,

157 rue de la Porte de

Trivaux,

92141 Clamart, France

E Peltier
\end{abstract}

Service de

Dermatologie,

Hôpital Henri Mondor,

51 Avenue du Maréchal

de Lattre de Tassigny,

94010 Créteil Cédex.

France

P Wolkenstein

\section{Service de}

Parasitologie

M Deniau

Service d'Anatomie et de Cytologie

Pathologiques

E-S Zafrani

J Wechsler

Correspondence to:

Janine Wechsler, MD.

Accepted for publication

1 June 1995

\section{Abstract}

A case of late stage cutaneous leishmaniasis with focal caseous necrosis is reported. The patient, a 30 year old Tunisian man, presented with idiopathic bone marrow aplasia. Microscopically, minimal changes were observed in the epidermis: slight hyperkeratosis and moderate acanthosis. Lesions predominated in the dermis. Epithelioid granulomas were found in the lower dermis. Some of these lesions were clearly surrounded by a ring of lymphocytes and were rarely confluent. $A$ peculiar histological feature was the presence of focal acidophilic and slightly granular necrosis at the centre of some the tuberculoid lesions. Focal fibrinoid necrosis was found in the upper dermis, outside granulomas. A mild to moderate infiltrate of histiocytes, lymphocytes and plasma cells, with scanty neutrophils, was observed mainly in the upper dermis. No intracellular or extracellular Leishman-Donovan bodies were observed. Acid fast mycobacteria, however, were not detected. Leish- maniasis was diagnosed on culture of skin biopsy specimens. The presence of caseous necrosis could lead to diagnostic confusion and result in an erroneous diagnosis of, for example, tuberculosis, syphilis, acne agminata, and sarcoidosis with fibrinoid necrosis. This is especially the case when parasites are scanty or absent.

(F Clin Pathol 1996;49:517-519)

Keywords: cutaneous leishmaniasis, caseous necrosis, pathology.

Cutaneous leishmaniasis, caused by infection with a flagellate protozoon, is endemic in the Mediterranean region. ${ }^{12}$ The disease can occur in one of four clinical forms: acute leishmaniasis, chronic leishmaniasis, leishmaniasis recidivans, and diffuse cutaneous leishmaniasis. ${ }^{13}$ Ninety per cent of cases of acute cutaneous leishmaniases resolve without treatment, healing with scar formation. ${ }^{2}$ The remaining cases generally evolve into chronic disease. ${ }^{45}$ 